\title{
Il ruolo dell'identità, del capitale sociale e delle certificazioni territoriali nello sviluppo locale dei territori minori: il caso di Pitigliano nella Regione Toscana
}

\author{
Viviana Calzati ${ }^{\text {i }}$ \\ Università di Perugia (Italy)
}

\begin{abstract}
Riassunto: L'obiettivo del presente paper è contribuire ad una maggiore comprensione delle relazione tra comunità locale, identità, capitale sociale e certificazioni territoriali. Nello specifico l'indagine si è riferita al modo con il quale gli abitanti del comune di Pitigliano percepiscono e valutano il loro borgo in termini di qualità, il ruolo che essi attribuiscono alle certificazioni territoriali e a quale elemento del centro urbano o del territorio si sentono maggiormente legati. Il borgo di Pitigliano è stato individuato come area di ricerca in quanto presenta caratteristiche riconducibili ai territori definiti "lenti" . In tali territori si individuano nuove traiettorie di sviluppo in cui l'identità, la storia locale, il capitale sociale, il patrimonio culturale, la qualità e la bellezza possono divenire innovativi fattori capaci di promuovere non un prodotto turistico, ma uno sviluppo endogeno del territorio in un'ottica di sostenibilità.
\end{abstract}

Palabras clave: Territori lenti; Identità; Comunità locale; Qualità; Certificazioni territoriali.

Title: The role of identity, social capital and territorial certifications in the local development of minor territories: the case of Pitigliano in the Region of Tuscany

\begin{abstract}
The objective of this paper is to further understanding of the relationship between local community, identity, social capital and territorial certifications. More specifically, the investigation deals with the way in which the inhabitants of the Municipality of Pitigliano perceive and evaluate their town in terms of quality, the role they attribute to territorial certifications and the elements of the town or the area with which they feel the strongest ties. The town of Pitigliano was identified as a research area because it has characteristics that include it among the territories defined as "slow." In these areas, new paths for development are identified in which identity, local history, social capital, cultural heritage, quality and beauty can become innovative factors that are able to promote not a tourist product, but rather an endogenous development of the area in a sustainable manner.
\end{abstract}

Keywords: Slow territories; Identity; Local community; Quality; Territorial certifications.

i Dipartimento di Discipline Giuridiche e Aziendali, Università di Perugia Italia. E-mail: viviana.calzati@progetti.unipg.it 


\section{Introduzione}

Il presente lavoro partendo da una nuova visione di territorio, che attribuisce valore alle identità, al capitale sociale e al patrimonio culturale autoctono, analizza la relazione tra comunità locale, identità e certificazioni territoriali.

In questo contesto il paper mira inizialmente a caratterizzare i principali tratti di un percorso di sviluppo sostenibile attraverso l'analisi di territori minori che nel presente lavoro vengono definiti lenti. Si evidenzia quindi un cambiamento radicale, rispetto ai tradizionali modelli di crescita esogena, che si allontana da una logica meramente economicistica in cui prevale il binomio sviluppo crescita economica. Nei territori lenti si individuano nuovi traiettorie di sviluppo in cui l'identità, la storia locale, il capitale sociale, il patrimonio culturale, la qualità e la bellezza possono divenire innovativi fattori strategici di sviluppo.

Successivamente particolare attenzione è rivolta alla nozione di identità locale e di capitale sociale evidenziando il legame stretto tra questi fattori in quanto entrambi fanno riferimento ad ambiti relazionali presenti su un determinato contesto territoriale. Inoltre tali fattori possono creare condizioni favorevoli a fenomeni in grado di generare innovazione in quanto il territorio non è solo luogo in cui si accumulano e sedimentano conoscenze ma in un ottica dinamica queste si rinnovano, si riproducono e si condividono.

Una parte del lavoro è dedicata al rapporto tra l'identità locale e le certificazioni territoriali per destinazioni al fine di evidenziare come le certificazioni possono divenire uno dei possibili strumenti di gestione del territorio attraverso cui promuovere sia un turismo di qualità sia la cultura dell'accoglienza.

Nell'ultima parte del lavoro si presentano i risultati di un indagine realizzata nel comune di Pitigliano piccolo borgo della Maremma toscana. L'indagine ha coinvolto i residenti con l'obiettivo di comprendere se nel piccolo borgo fattori quali l'identità, la qualità e la bellezza sono percepiti e ritenuti dalla comunità locale un bene comune da tutelare e valorizzare in un ottica di sviluppo sostenibile endogeno e durevole.

\section{Territori lenti e identità locale}

I territori vivono attualmente nelle proprie dinamiche di sviluppo e di competitività una fase di profondo mutamento. I territori minori ritenuti ai margini dei sistemi forti e consolidati individuano nel territorio un fattore competitivo importante in cui si rafforzano interessi e identità collettivi e in cui la sua coesione sociale rappresenta valore aggiunto capace di promuovere distretti di qualità. Oggi viviamo una fase di ri-personalizzazione dell'economia e quindi anche dello spazio fisico, che pone al centro i significati elaborati dalle persone e conferiti ai luoghi (Bonomi e Rullani, 2005).
In anni recenti, diversi territori hanno attivato percorsi di sviluppo locale attraverso la valorizzazione delle identità locali, l'attenzione per l'ambiente e il paesaggio e la capacità dei beni culturali e dei prodotti enogastronomici di raccontare la storia e la tradizione dei luoghi. Il tratto comune di questi territori è la presenza di un'alta qualità della vita e del paesaggio che, unita a cultura, tradizioni, storia locale ma anche tecnologia e ricerca, può dare vita a nuovi "distretti culturali"(Calzati, 2009).

Tali territori definiti lenti non evidenziano una situazione di ritardo o di arretratezza ma semplicemente di funzionare con un motore a basso numero di giri in cui la lentezza costituisce il fattore distintivo di sviluppo non centrato sul concetto di crescita (Lancerini, Lanzani, 2005). Pertanto è possibile in alcune aree individuare percorsi di sviluppo in cui al centro non vi è unicamente la capacità e la forza di un sistema economico produttivo di generare reddito ma risulta importante considerare altre forme di patrimonio, di capitale e di strumenti per la produzione del reddito e di valore in una logica che implica il ripensamento del mito della crescita. I territori lenti sono aree a bassa densità demografica, caratterizzate da un contesto rurale che evidenzia ancora una forte presenza dell'agricoltura, in cui il patrimonio storico-artistico è spesso poco noto, ma di qualità. Il modello di ospitalità che caratterizza tali territori è composto da strutture integrate con il paesaggio locale (bed and breakfast, agriturismo, case per vacanza) e le attività culturali sono orientate alla valorizzazione delle tradizioni popolari, della storia e dell'identità locale. Nei territori lenti si configurano modelli di sviluppo, diversi da quelli tradizionalmente intesi, in cui si mescolano attività agricole, turismo innovativo e attività produttive originali che evidenziano un'ottica plurisettoriale e quindi l'assenza di un unico motore di sviluppo

In anni recenti è il concetto stesso di crescita ad essere messo in discussione in quanto troppo spesso utilizzato come sinonimo di "sviluppo". Il concetto di sviluppo è più ampio e omnicomprensivo di quello di "crescita", in quanto coinvolge aspetti culturali ed etici che lo riconducono a quello di miglioramento di qualità della vita. In tale contesto si inserisce il dibattito in corso sul tentativo di individuare nuovi parametri capaci di misurare beni intangibili quali la coesione sociale, la qualità, la felicità con l'obiettivo di individuare un modello di sviluppo caratterizzato non dalla ricerca di un continuo aumento dei PIL ma dalla diffusione della qualità in tutti i settori economici, evidenziando così una nuova logica su cui fondare il sistema socio-economico. Quanto esposto implica un cambio di direzione e l'adozione di una valutazione multicriteriale dell'economia, in quanto la natura essenzialmente multidimensionale dello sviluppo sostenibile, comprendente aspetti economici, ambientali, sociali ed istituzionali e richiede quindi l'adozione di misure che rappresentino i vari aspetti di sviluppo sostenibile nel tempo (Shmelev e Rodriguez-Labajos, 2009).

In questa visione multidimensionale dello sviluppo una spinta teorica importante è costituita dall'approccio 
delle capabilities di Amartya Sen (1999), dalla ricerca sulla felicità di Layard (2006), dai potenziali collegamenti tra lo studio dell'economia e quello della psicologia di Daniel Kahneman e Alan Krueger (2006) sul National Wellbeing Account e dal recente Rapporto della cosiddetta "Commissione Stiglitz" sulla misura delle prestazioni economiche e del progresso sociale (2009).

Solo recentemente le tradizionali contrapposizioni crescita/decrescita limite/sviluppo sono, con l'avvio del ciclo della green e soft economy, nuova forma di modernità, superate e incorporate.

In questo rinnovato contesto anche i territori lenti, attraverso un percorso capace di miscelare forme diverse di innovazione, culturale, sociale, tecnica, di partecipazione e ambientale con una visione condivisa, endogena e comunitaria, possono uscire dai margini e posizionarsi come "distinct areas" orientate a promuovere uno sviluppo sostenibile (Calzati 2011)

Nei territori lenti e più in generale per lo sviluppo locale, si evidenzia il ruolo importante e integrativo di fattori immateriali quali l'identità locale, le reti, il capitale sociale e la governance (Mutti 1998, Pichierri 1995, Trigilia, 1999) in grado di originare, se adeguatamente valorizzati, percorsi innovativi di sviluppo turistico locale.

Infatti in anni recenti è iniziato un nuovo processo orientato a ricostruire nuovamente una identità culturale locale intesa come individuazione, tutela e valorizzazione di tutti i fattori che contribuiscono a creare la specificità e l'unicità dei luoghi e dell'ambiente.

Occorre evidenziare come nei paesi anglosassoni il "local heritage" abbia dato origine ad un percorso più maturo e a un dibattito più ampio in cui lo heritage ha assunto grande valore come ricerca approfondita sulla storia e sulla cultura dei singoli territori in un ottica non solo di tutela, conservazione e valorizzazione ma come fattore in grado di promuovere sviluppo economico e sociale in aree minori.

Il termine heritage è utilizzato con un significato ampio che implica varie dimensioni dello stesso (Tunbridge e Ashworth 1996) e conduce a considerare lo heritage come qualunque tipo di scambio o relazione intergenerazionale (Graham et al. 2000). Pertanto creando un unicum tra storia, cultura, e paese in cui le persone vivono, emerge una gamma di luoghi dell' heritage che comprende una insieme di elementi tangibili o intangibili (Boyd 2003):

- $\quad$ edifici e monumenti storici;

- siti di importanti avvenimenti passati, quali le battaglie;

- $\quad$ paesaggio tradizionale e flora e fauna locali;

- lingua, letteratura, musica e arte;

- $\quad$ eventi tradizionali e pratiche popolari;

- $\quad$ stili di vita tradizionali, tra cui cibi, bevande e sport.

Tale definizione di Boyd sostenuta anche da Mc Kercher e du Cros (2002) evidenzia come le traiettorie di sviluppo dei territori minori siano fortemente connesse con elementi intangibili, maggiormente rappresentativi dell'identità di una comunità locale.

L'identità ${ }^{1}$ locale è intesa in una duplice accezione: come percezione e rappresentazione di sé da parte degli attori locali e come un luogo viene percepito dell'esterno. La prima concorre alla costruzione della vision di un territorio la seconda assume un ruolo fondamentale nella valorizzazione turistica di un luogo.

I dati più recenti sui comportamenti turistici degli europei confermano come l'identità delle località visitate risulti un elemento chiave nella scelta delle destinazioni, attuata nella maggioranza dei casi (32\%) sulla base dell'ambiente locale inteso nell'accezione di attrattività globale dei luoghi (Commissione Europea, 2010).

In un percorso di valorizzazione del territorio elementi intangibili quali l'identità, il patrimonio culturale e il capitale sociale costituiscono la matrice identitaria e l'armatura culturale del territorio (Carta,2002) su cui attivare innovative dinamiche di sviluppo locale.

\section{Capitale sociale e innovazione possibile leva compe- titiva nei territori lenti}

Negli ultimi decenni, come già evidenziato, la letteratura economica ha rivolto sempre maggiore attenzione ai fattori immateriali e relazionali per spiegare lo sviluppo e la crescita economica. In questo rinnovato contesto, in cui la relazionalità emerge come fattore rilevante, la fiducia, il senso di appartenenza, la creatività e l'identità locale diventano elementi essenziali per la competitività locale.

Il concetto di capitale sociale è stato introdotto da Jacobs (1961) e definito con maggiore precisione da Coleman (1990) che lo utilizza per indicare una risorsa che non risiede né negli individui, né nei mezzi di produzione ma nella struttura delle relazioni sociali. Il capitale sociale è perciò una risorsa collettiva, è connesso con la struttura delle reti di relazioni, con la loro densità e con la stabilità delle relazioni stesse nel tempo. Coleman fa anche riferimento alle ricerche di sociologia economica di Granovetter (1985) in cui si introduce il concetto di embeddedness (radicamento) in riferimento alla struttura sociale dell'azione economica che è influenzata dalle relazioni personali degli individui e dal complesso generale di interazioni tra le relazioni stesse. Il concetto di capitale sociale come civicness è introdotto da Putman (1993) che lo definisce come quell'insieme di elementi dell'organizzazione sociale, quali la fiducia, la solidarietà, le norme di reciprocità, le reti di associazionismo civico, in grado di migliorare l'efficienza della società promuovendo il coordinamento delle azioni individuali e l'azione collettiva. Fukuyama (1995-1996) lega le origini del capitale sociale alla storia e alle tradizioni locali e la sua definizione al concetto di fiducia e di condivisione di sistemi valoriali collettivi.

Nella letteratura italiana il capitale sociale viene definito come sedimentazione di ciò che viene investito nelle strutture relazionali fra individui e organizzazioni. Si tratta di beni collettivi relazionali o reti tra gli attori che consentono la diffusione di conoscenze e informazioni e riducono i loro costi di diffusione favorendo la capacità di coordinamento. Trigilia (1999) evidenzia come attraverso il capitale di relazioni si rendono disponibili "risorse cognitive, come le informazioni, o normative, come la fiducia, 
che permettono agli attori di realizzare obiettivi che non sarebbero altrimenti raggiungibili, o lo sarebbero a costi molto più alti" (Trigilia, 1999: 423). Mutti (1998) definisce il capitale sociale come una struttura di relazioni tra persone durevole nel tempo in grado di favorire la cooperazione e produrre quindi valori materiali e simbolici e sono le reti fiduciarie formali e informali che stimolano la cooperazione e la reciprocità. Bagnasco (2001) propone una distinzione del capitale sociale tra una concezione "sistemica" o "culturalistica" e una concezione "relazionale" o "interattiva". La prima fa riferimento al capitale sociale inteso come l'attitudine a cooperare che ha origine da una cultura cooperativa condivisa, in grado di creare fiducia interpersonale diffusa. La seconda individua il capitale sociale nelle risorse per l'azione che scaturiscono dal tessuto di relazioni cooperative in cui un individuo è inserito.

Il territorio non è solo luogo in cui si accumulano e sedimentano conoscenze ma in un ottica dinamica queste si rinnovano, si riproducono, si condividono e si scambiano creando condizioni favorevoli a fenomeni in grado di generare innovazione. Il territorio diviene quindi luogo di innovazione in quanto capace di trasformare e moltiplicare la conoscenza sedimentata da fattori storici, ma costantemente riprodotta e potenziata dalle innovazioni dei soggetti che lo abitano (Rullani 2003). Occorre evidenziare come l'innovazione sia sempre stata considerata un fenomeno urbano in quanto è in tale contesto che sono presenti gli attori sociali innovatori, che si crea contaminazione culturale e che si diffondono più rapidamente le innovazioni tra la sfera culturale, scientifica e produttiva. Pertanto i territori ai margini delle aree urbane (rurali piuttosto che alpini) sono stati sempre considerati aree avverse all'innovazione a causa della scarsità delle risorse economiche e dell'arretratezza culturale degli attori economici, sociali e politici. Nella nuova visione post-fordista la maggiore diffusione degli strumenti culturali dovuta alle nuove tecnologie della comunicazione e il ruolo del territorio come luogo delle differenze, dove le reti si intrecciano e le esperienze si contaminano, contribuiscono a rimuovere o ad attenuare alcuni dei vincoli che conducevano ad identificare le aree marginali come luoghi della tradizione ostili all'innovazione.

In tale contesto la creatività è un input fondamentale per la produzione di cultura a condizione che sia orientata da valori etici e culturali e diventi strumento per conseguire obiettivi dotati di valore e socialmente condivisi. In Italia migliaia di città storiche molti distretti industriali e parte delle nostre innovazioni tecnologiche hanno una lunga tradizione di accumulazione di conoscenze ed esperienze (Santagata, 2009).

La sfida dei territori minori è quella di trovare un equilibrio capace di sviluppare e riconoscere il valore della cultura, ma anche di garantire le condizioni necessarie per la continua rigenerazione del sistema socio-economico.

In tale contesto i territori lenti danno origine ad un modello di sviluppo originale fondato sul binomio tradizione e innovazione in un approccio distrettuale ricon- ducibile a distretti culturali evoluti, in cui il territorio con la sua storia, tradizione, saperi e identità relazionale costituisce un valore aggiunto e un vantaggio competitivo difficilmente riproducibile in altri contesti.

\section{Identità locale e certificazioni territoriali: possibile binomio per uno sviluppo turistico sostenibile}

Nei territori lenti portatori di una nuova visione del territorio come valore in cui è presente il distacco tra benessere e crescita economica l'identità locale costituisce un elemento del prodotto turistico del territorio che come già evidenziato si trasforma e si rinnova attraverso i processi di modernizzazione.

Il territorio si definisce per la sua identità, ossia l'insieme di tutte quelle componenti costruite attorno ad un progetto di trasformazione continua e di sviluppo nel tempo ad opera di chi lo vive (Maggi-Falletti, 2000).

Esso esprime un ruolo attivo dello sviluppo in quanto solo nel territorio si evidenziano dei particolari fattori storico,culturali e sociali che sono alla base dei modelli di organizzazione della produzione e quindi dei processi di mutamento economico e sociale dell'area stessa (Garofoli 1994). Il territorio diviene luogo dove hanno origine relazioni e si scambiano informazioni e conoscenze; gli ambiti locali rivestono, quindi, un ruolo importante nel determinare le caratteristiche e la qualità dello sviluppo. (Capuano, 2007).

La valorizzazione dei territori minori e del loro patrimonio culturale ${ }^{2}$ materiale ed immateriale pone in evidenza il legame tra heritage e turismo. Il modello di sviluppo turistico dei territori lenti evidenzia l'importanza del patrimonio culturale costituito, non solo dalle sue forme materiali, ma anche da attività culturali quali feste religiose, manifestazioni folkloristiche ecc. espressione della tradizione e della produzione contemporanea. Richards (2001) considera la cultura costituita di processi (idee, stile di vita ecc.) ed effetti di tali processi (edifici, costumi, prodotti, arte ecc.) in quanto il turismo culturale non è solo la visita di siti e monumenti ma comprende la fruizione dei modi di vita dei luoghi visitati

Nei territori minori si evidenzia, da un lato, la necessità di fare sistema al fine di superare una connotazione settoriale che ha limitato le potenzialità di sviluppo del turismo italiano e dall'altro l'importanza del coinvolgimento della comunità locale che interagisce con gli attori istituzionali e gli operatori del settore. Si stabilisce una specie di patto sociale che nel promuovere l'accoglienza del turista contestualmente promuove la qualità della vita dei residenti. I territori lenti individuano nella comunità locale un capitale sociale in grado: di condividere obiettivi e valori, di collaborare al fine di definire un'offerta turistica differenziata, di essere soprattutto spinta concreta allo sviluppo (Mazzoli, 2007). Tali territori che nelle loro traiettorie di sviluppo si caratterizzano per la loro identità, unicità, specificità e differenza rispondono all'attuale domanda di consumo turistico e di vacanza che si traduce 
in un vero e proprio "stile di consumo" orientato principalmente all'incontro "reale" con la comunità locale. Infatti, il turista desidera non visitare ma entrare in contatto con le tradizioni, abitudini, costumi, patrimonio culturale e ambientale e con la comunità che li produce. A fronte di questa interazione fra il turista e il territorio, quest'ultimo deve rispondere con una gestione integrata delle risorse e con l'attivazione di politiche bottom up in cui gioca un ruolo determinante la cooperazione e concertazione degli attori locali capaci di riconoscersi in valori condivisi.

La vision di tali destinazioni ha origine dalla composizione delle visioni strategiche di numerosi operatori, il cui grado di coinvolgimento nell'offerta turistica è differente ed implica una richiesta di partecipazione alla pianificazione delle proposte turistiche da parte della popolazione residente nella comunità al fine di conoscere e controllare il futuro del proprio territorio (Ritchie 1993).

Nella gestione di una destinazione le certificazioni e i marchi di qualità rappresentano elementi di "amplificazione" per contesti territoriali orientati allo sviluppo turistico. In uno scenario in cui la competizione tra destinazioni turistiche è sempre più forte assumono un ruolo decisivo da un lato la qualità delle strutture, delle risorse e delle attrazioni e dall'altro la gestione, le politiche di sviluppo e di pianificazione. All'interno del destination marketing, riconosciuto in letteratura come uno dei fattori fondamentali per la competitività della destinazione (Keller, 1998; Buhalis, 2000); Prideaux e Cooper, 2003; Kotler, Bowen e Makens, 2007; Martini e Ejarque, 2008), ha assunto recentemente un ruolo rilevante l'applicazione del brand management al territorio (Blain, Levy e Ritchie (2005); Kavarantizis (2005); Martini (2005); Golinelli e Simoni (2006). Il brand territoriale si distingue da un brand aziendale in quanto caratterizzato da proprie specificità legate al fatto di essere attribuito non a un prodotto/ servizio ma a un intero territorio costituito dalla presenza di risorse e attori che sono coinvolti nella definizione dell'offerta turistica.

Le certificazioni per destinazioni possono essere suddivise in tre categorie: le label, i sistemi di gestione certificati e i premi (Carbone, Fava, Maggi, 2000).

Le certificazioni territoriali, intese come strumenti volontari in grado di promuovere le risorse del territorio e un turismo di qualità, devono essere sostenute da altri strumenti di gestione sia di carattere economico che normativo, in quanto da sole non sono in grado di garantire uno sviluppo turistico sostenibile.

L'ottenimento della certificazione diventa per i comuni possibilità di presentare un'offerta aggiuntiva contribuendo ad avviare percorsi di sviluppo orientati alla qualità, in quanto l'adesione a tali marchi comporta il rispetto di requisiti, talvolta anche strutturali, molto rigorosi. Coerentemente i piccoli comuni possono contribuire a realizzare progetti di sostenibilità ambientale, turistica e sociale, grazie al rafforzamento delle produzioni locali (ad esempio potenziamento delle coltivazioni biologiche, richiesta di marchi di tutela) ed alla valorizzazione delle tradizioni (tutela dell'artigianato, delle produzioni tipiche).
Le certificazioni per destinazioni rappresentano uno dei possibili strumenti di gestione del territorio attraverso cui promuovere sia un turismo di qualità, fondato sulla valorizzazione delle risorse locali, dell'artigianato, delle produzioni tipiche e dell'identità, sia per implementare la cultura dell'accoglienza, al fine di perseguire uno sviluppo economico e sociale in un' ottica sostenibile.

\section{Pitigliano come i residenti percepiscono il borgo}

L'attività di indagine e di rilevazione è stata effettuata nel comune di Pitigliano. Il borgo di Pitigliano è stato individuato come area di ricerca in quanto territorio capace di attivare percorsi di sviluppo locale agendo sul recupero e sulla valorizzazione delle identità locali, sull'attenzione per l'ambiente e l'arredo urbano, sulla capacità dei beni culturali e dei prodotti enogastronomici di narrare la sua storia e le sue tradizioni. Le numerose certificazioni conferite a Pitigliano attestano l'attenzione e l'importanza che il piccolo borgo attribuisce alla qualità, alla bellezza e all'identità infatti il comune è Bandiera arancione, Borgo più bello d'Italia, Città del Vino, Città dell'Olio e Città del Tufo.

La ricerca mira a comprendere, attraverso la somministrazione di 131 questionari rivolti ai residenti nel periodo pre-natalizio, come i cittadini percepiscono e valutano il loro borgo in termini di qualità, il ruolo che essi attribuiscono alle certificazioni territoriali e quali interventi ritengono opportuni al fine di migliorare la qualità della vita e la competitività turistica del territorio. Occorre evidenziare che si tratta di un'indagine esplorativa, su un campione non statisticamente rappresentativo del comune di Pitigliano, comunque in grado di elaborare elementi di riflessione interessanti circa i legami tra qualità. identità, certificazioni e territorio che potrebbero essere approfonditi al fine di fornire indicazioni utili, agli amministratori e operatori del territorio, in grado non solo di incrementate ma di valorizzare e "diffondere" la qualità del borgo e quindi la sua visibilità turistica. Si è ritenuto opportuno realizzare una mappatura delle risorse culturali del comune, utile a comprendere come Pitigliano rappresenti il tipico borgo italiano ricco di storia locale, patrimonio culturale tangibile e intangibile capace non solo di attrarre turisti ma grazie alla sua bellezza e qualità essere luogo del "buon vivere" in primis per la comunità locale.

\section{Analisi dell'offerta delle risorse culturali del terri- torio di Pitigliano}

L' "Area del Tufo", che prende il nome dalla ricca presenza di rocce tufacee, è la parte meridionale della Maremma, al suo interno nella parte sud orientale della Provincia di Grosseto si trova il comune di Pitigliano. Il territorio comunale si estende su una superficie di $102,89 \mathrm{~km}^{2}$ e conta 3.971 abitanti, con una densità di 39 abitanti per $\mathrm{km}^{2}$. 
Da una ricerca realizzata da Doxa, Mercury e Borghi autentici d'Italia, finalizzata a un'indagine sui visitatori attuali e potenziali dei borghi caratteristici, ${ }^{3}$ emerge la motivazione che spinge i turisti verso i borghi italiani. Infatti la ricerca indica le principali motivazioni: nella scoperta di posti nuovi o poco conosciuti, nella tranquillità e serenità dei luoghi, nella scoperta di tradizioni gastronomiche e prodotti tipici regionali, nella convivialità degli abitanti del posto e nell'appagamento dei sensi. Lo studio sottolinea come il borgo italiano accanto a determinate caratteristiche fisiche (luogo poco conosciuto, dimensione legata al passato, un elemento di attrazione e immerso in una natura tipica della zona), deve possedere qualità relazionali ed emozionali insite nelle comunità che abitano i borghi e proprie dei ritmi e tempi di vita lenti. Inoltre emerge nei piccoli centri la carenza di strutture ricettive, di servizi di accoglienza e una comunicazione priva di punti di riferimento chiari e univoci elementi che penalizzano le prospettive di crescita dei borghi e la loro valorizzazione turistica.

Il comune di Pitigliano ben si identifica in questo profilo sia per quanto riguarda le caratteristiche fisiche e la qualità

del capitale sociale e si pone come meta competitiva per quel settore della domanda che cerca un immersione nel territorio, un'esperienza esclusiva e unica in un ottica di turismo esperienziale (Pine e Gimore, 2000).

Il territorio del comune di Pitigliano risulta caratterizzato da numerose testimonianze di carattere storico artistico e religioso, da un patrimonio naturalistico-ambientale di qualità e da un patrimonio culturale intangibile in un accezione ampia del termine heritage (Boyd 2003), come già evidenziato, che comprende tradizioni, artigianato, enogastronomia, eventi culturali e feste religiose. In questo contesto si inserisce la ricognizione delle principali risorse del patrimonio culturale e ambientale inteso come parte integrante di un sistema complesso di coordinamento orientato alla valorizzazione dell'identità del territorio sia al suo interno che verso l'esterno.

La metodologia di ricognizione delle principali risorse del comune di Pitigliano ha condotto alla suddivisione degli attrattori in 4 categorie: la cultura, l'ambiente, le reti e le iniziative intercomunali e le associazioni e i marchi di qualità. Occorre evidenziare come il lavoro che segue è orientato a far emergere, oltre alle risorse locali (cultura e ambiente) anche un aspetto ancora poco indagato nelle attività di mappatura, quale la presenza di reti e di marchi di qualità, che dovrebbero costituire valore aggiunto in quanto non semplici strumenti di promozione, ma veri e propri attivatori di sistema. Da un'analisi delle diverse tipologie di attrattori turistici, suddivisi in quattro cate- gorie: la cultura, l' ambiente, le reti e le iniziative intercomunali e le associazioni e i marchi di qualità, risulta evidente il ruolo fondamentale ricoperto dalla numerosa presenza di beni storico-architettonico-archeologici (48), forte espressione della storia e delle dinamiche sociali di Pitigliano, ad oggi conosciuta anche come "The Little Jerusalem" per la storica presenza di rappresentanti della comunità ebraica, che per secoli ha influenzato di riflesso l'architettura del luogo con la nascita di Sinagoghe e altri luoghi di culto. (Figura 1). ${ }^{4}$

Numero attrattori per tipologia

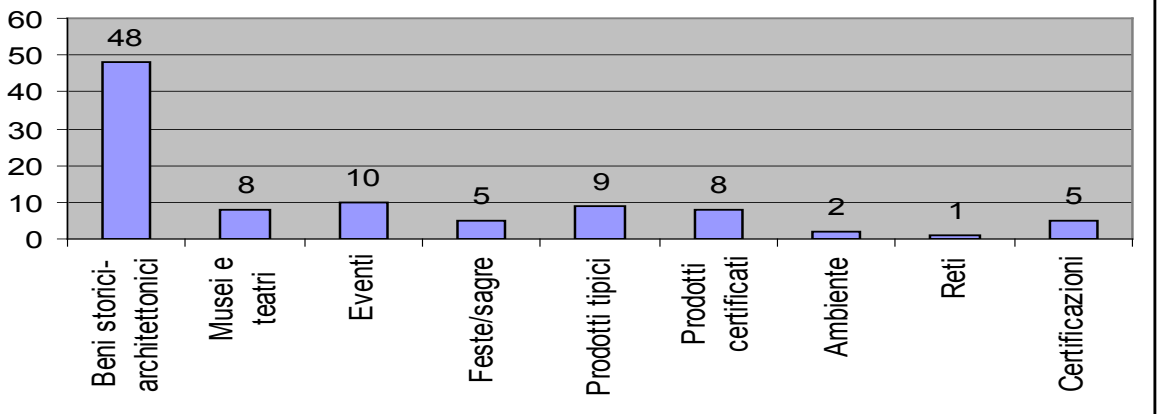

Figura 1 Numero degli attrattori per tipologia. Fonte: ns elaborazione

Ai fini del presente lavoro particolare attenzione è stata rivolta alle certificazioni e marchi di qualità ottenuti dal comune. Il borgo di Pitigliano ha ottenuto il marchio di Bandiera Arancione marchio di qualità turistico-ambientale conferito da Touring-Club Italiano, e destinato a piccoli comuni che, come Pitigliano, si caratterizzano per un'offerta di eccellenza e un'accoglienza di qualità. Il conseguimento di tale certificazione presuppone una politica di valorizzazione delle risorse locali e uno sviluppo della cultura dell'accoglienza in un'ottica di rafforzamento dell'identità territoriale. Pitigliano inoltre aderisce al club di prodotto "I Borghi più belli di Italia", certificazione conferita a quelle realtà comunali che rispettano requisiti come l'armonia architettonica del tessuto urbano, la qualità del patrimonio edilizio pubblico e privato e che in generale attengono alla vivibilità del borgo in termini di attività e di servizi al cittadino. Il comune oggetto di analisi aderisce alle Associazioni Nazionali di "Città dell'Olio" e di "Città del Vino", in riferimento alla sua produzione di vino $\mathrm{DOC}^{5}$ e di olio IGP. Pitigliano è "Città del Tufo" insieme alle località limitrofe di Sovana e Sorano, in quanto tutto il territorio è di origine vulcanica ed è frastagliato da enormi speroni tufacei. Occorre evidenziare come tutte le certificazioni ottenute dal comune di Pitigliano aderiscono al marchio ombrello RES Tipica ${ }^{6}$ a conferma della volontà dell'amministrazione locale di promuovere un modello di crescita orientato ad incrementare la qualità della vita, la messa in valore delle specificità e il connubio tra produzio- 
ni e identità locali al fine di promuovere uno sviluppo turistico sostenibile.

\section{Il questionario}

Il territorio di Pitigliano, come già evidenziato, presenta caratteristiche riconducibili ai territori lenti la cui lentezza non è indice di ritardo, ma fattore distintivo di sviluppo, dove la qualità del patrimonio culturale, del paesaggio e della vita diventa un bene comune della comunità. Pertanto si è voluto indagare, attraverso la somministrazione di un questionario, come i residenti percepiscono e valutano il loro borgo al fine di comprendere se hanno consapevolezza di abitare e vivere in un territorio di qualità. Il questionario è costituito da quattro diverse sezioni tematiche:

1. Profilo del cittadino intervistato: la prima area d'indagine orientata a tracciare un profilo del cittadino intervistato (sesso, età, titolo di studio, professione)

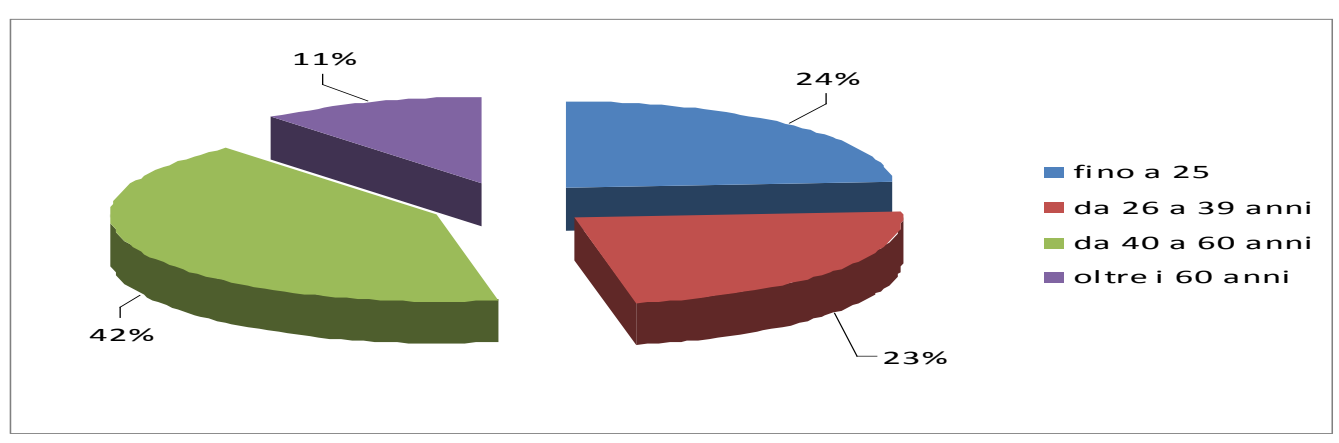

2. Rapporto residenti territorio:

nella seconda area si vuole analizzare la motivazione che spinge il cittadino a continuare a risiedere nel comune, individuare gli elementi che rappresentano secondo i residenti l'identità di Pitigliano e l'elemento del territorio al quale i residenti si sentono maggiormente legati

3. Valutazione dei residenti nei confronti delle certificazioni: una sezione del questionario è stata dedicata al tema delle certificazioni territoriali. Temi oggetto di indagine: la conoscenza da parte dei residenti delle certificazioni conferite al comune, l'individuazione di quale fattore concorre all'ottenimento della certificazione e la valutazio-

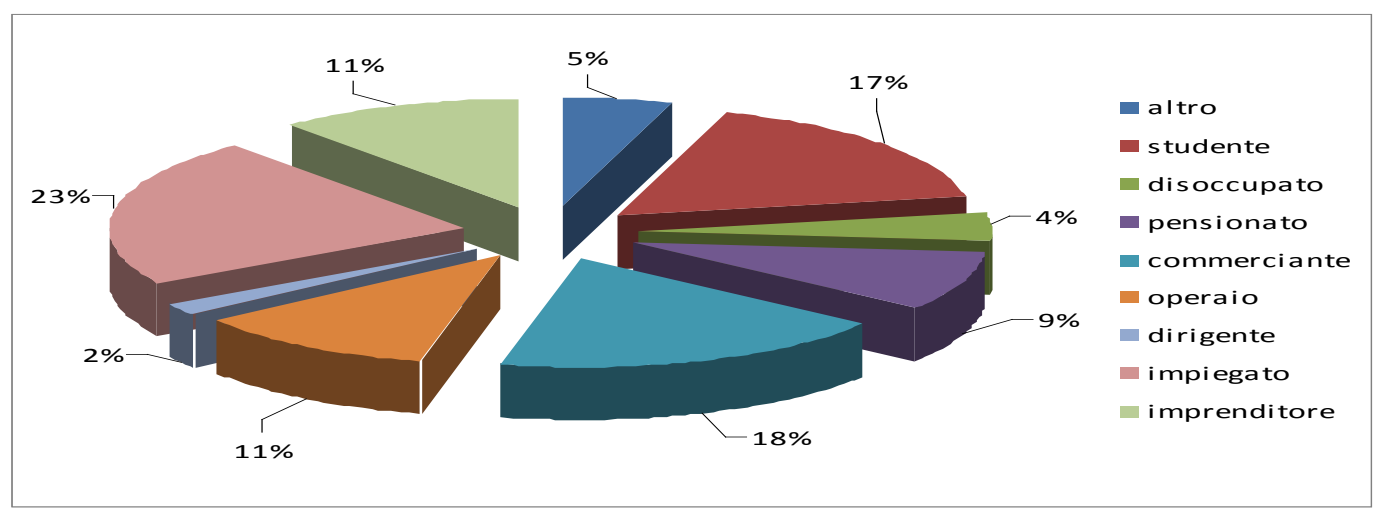

Figura 3 I settori di occupazione. Fonte: ns. elaborazione ne da parte dei residenti degli effetti delle certificazioni sul territorio

4. Qualità del territorio e turismo: con questa area del questionario si chiede ai residenti di esprimere il proprio grado di soddisfazione nei confronti dei provvedimenti comunali orientati alla valorizzazione del borgo. Inoltre si chiede di indicare in quali aree l'amministrazione dovrebbe intervenire al fine di implementarne la valorizzazione e quali interventi dovrebbe attuare per rendere Pitigliano una destinazione turistica maggiormente competitiva. In particolare una domanda è dedicata ad indicare in quali ambiti l'amministrazione locale dovrebbe implementare le risorse al fine di migliorare la qualità del territorio.
Sono stati somministrati 131 questionari nel periodo pre-natalizio (dicembre 2010). Il campione è stato selezionato seguendo un campionamento di tipo non probabilistico, intervistando in modo casuale i residenti su tutto il comune di Pitigliano. Le interviste sono state realizzate sia nel centro storico che nella parte nuova del borgo.

\section{Risultati dell'indagine \\ Il profilo dei residenti}

Le domande della prima sezione hanno delineato il profilo del residente intervistato. Con riferimento al sesso, si rileva che il $55 \%$ del campione è rappresentato 
da residenti di sesso maschile. Premesso che si è deciso di non intervistare residenti aventi un'età inferiore ai 18 anni, il maggior numero degli intervistati $(42 \%)$ ha un'età compresa tra i 40 e i 60 anni. Il $24 \%$ tra i 18 ed i 25 anni, il $23 \%$ tra i 26 e 39 anni. L'11\% ha un'età superiore ai 60 anni. (Figura 2)

Il $47 \%$ dei residenti ha conseguito un titolo di scuola media superiore a conferma di un livello di istruzione piuttosto elevato, il 40\% la licenza media inferiore, l'8\% la laurea e il $5 \%$ non ha proseguito il percorso di formazione dopo la licenza elementare.

L'analisi dei dati riguardanti il settore di occupazione (figura 3) evidenzia come gli impiegati sono la categoria più rappresentata $(23 \%)$, seguita da commercianti $(18 \%)$ e studenti (17\%).

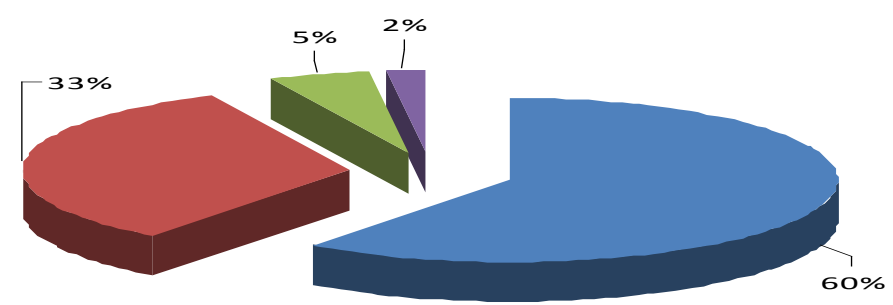

- dalla nascita

- più di 3 anni

da 1 a 3 anni

- meno di 6 mesi

Figura 4 Periodo residenza. Fonte: ns elaborazione

siedere nel borgo ha volutamente coinvolto anche coloro che lavorano in ambito esterno al comune ma continuano a risiedere a Pitigliano. Il 54\% dei residenti individua nelle relazioni sociali e familiari il motivo principale per il quale si continua a risiedere nel comune. Seguono la tranquillità del luogo (19\%) e la qualità della vita (15\%). Solamente il $4 \%$ degli intervistati individua nel basso costo delle unità immobiliari il motivo determinante nella scelta del luogo di residenza (figura 5). Tali dati evidenziano il ruolo fondamentale delle relazioni sociali e della qualità

\section{Il rapporto residen- ti territorio}

Nella seconda sezione del questionario è stato indagato il rapporto tra i residenti e il loro territorio, con riferimento al periodo di residenza e alle motivazioni che spingono il cittadino a continuare a risiedere nel comune. Particolare attenzione è rivolta a cogliere il legame tra comunità locale e identità, infatti è chiesto ai residenti di individuare quale elemento rappresenta maggiormente l'identità di Pitigliano e a quale elemento del territorio si sentono particolarmente legati.

Il $60 \%$ degli intervistati risiede dalla nascita nel comune di Pitigliano mentre il $33 \%$ vi risiede da più di 3 anni (figura 4). Tale dato evidenzia come la maggior parte dei residenti è legato al territorio sin dalla nascita e ciò rimanda al forte sentimento di appartenenza alla famiglia, la quale si è sempre caratterizzata come fenomeno sociale centrale, da cui si irradiano valori, credenze e tradizioni. Accanto alla soddisfazione dei bisogni umani fondamentali essa ha coordinato i rapporti economici, ha garantito ruoli, valori, funzioni sociali precise ai propri membri.

Nonostante la maggior parte degli intervistati lavori all'interno del comune (63\% contro il 37\%), l'analisi delle motivazioni per cui residenti pitiglianesi continuano a ri-

della vita del territorio a conferma che esso non è esprimibile solo in termini di fattori o dotazioni infrastrutturali. Il territorio al contrario si caratterizza come luogo in cui i valori sociali, una cultura condivisa, un'identità locale e il senso di appartenenza alla comunità diventano caratteri distintivi in grado di creare valore aggiunto.

Per quanto riguarda l'elemento che rappresenta maggiormente l'identità di Pitigliano (figura 6 ), il 26\% dei residenti individua tale elemento nel centro storico, il $23 \%$ nell'ambiente e paesaggio e il 18\% nel patrimonio storicoarchitettonico. Occorre evidenziare come solo il $12 \%$ individua nelle relazioni familiari e umane l'elemento che rappresenta maggiormente l'identità del borgo a conferma che i residenti collegano l'identità con aspetti tangibili e materiali del territorio anche se è nel centro storico che si sono sviluppate le prime relazioni sociali che hanno continuato a stratificarsi e consolidarsi nel tempo. 


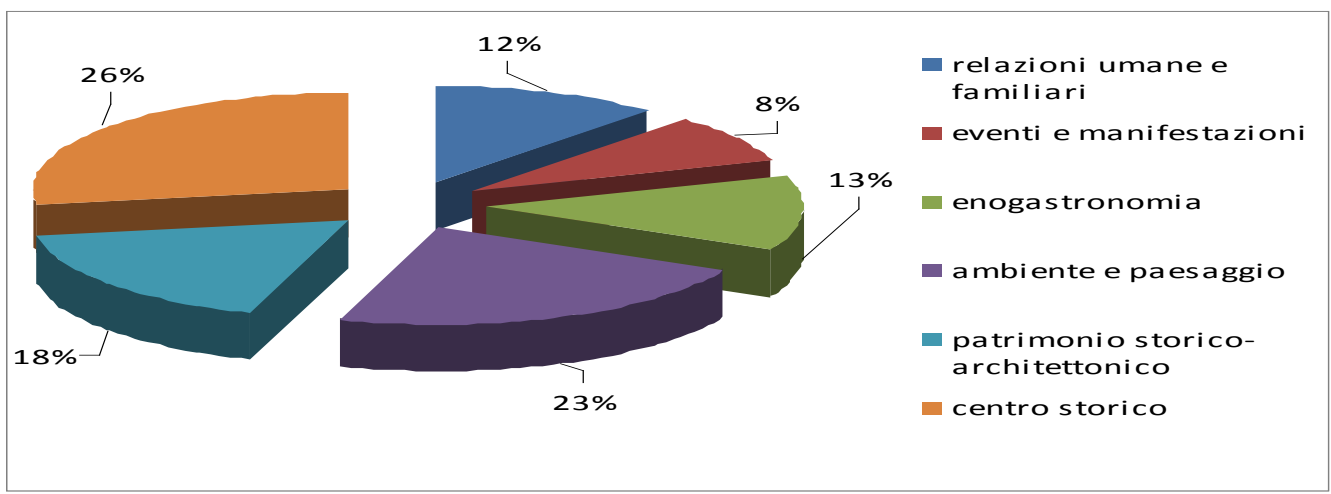

Figura 6 Elemento che rappresenta maggiormente l'identità di Pitigliano. Fonte: ns elaborazione

In riferimento al legame tra territorio e residenti, il $40 \%$ degli intervistati ha individuato nella bellezza del paesaggio l'elemento del territorio al quale si sente particolarmente legato. Il $20 \%$ dei residenti individua tale elemento nel patrimonio storico-architettonico (figura 7). Tali dati evidenziano due aspetti importanti del territorio:

\section{Le certificazioni territoriali: valutazione dei resi- denti}

Un'area di indagine del questionario è stata studiata per descrivere la conoscenza e la valutazione dei residenti rispetto alle certificazioni territoriali. L'85\% degli

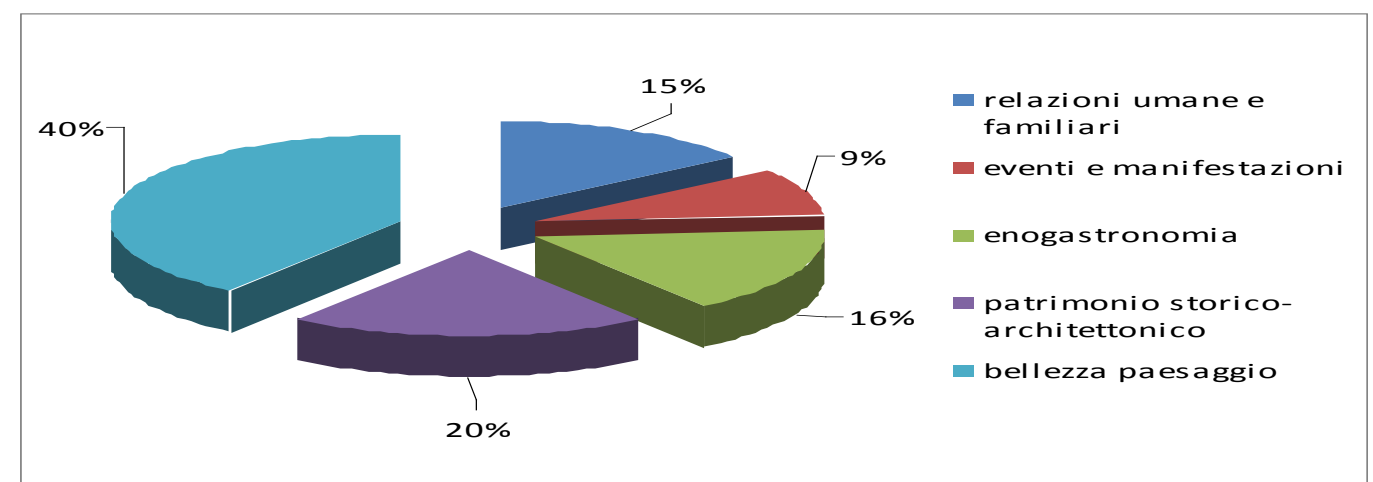

Figura 7 Elemento del territorio al quale i residenti si sentono maggiormente legati. Fonte: ns elaborazione intervistati è a conoscenza delle certificazioni conferite a Pitigliano (figura 8). La domanda prevedeva successivamente di selezionare tra un elenco (Borghi più belli d'Italia, Bandiera Arancione, Strada del Vino, Città dell'Olio, Città del Tufo, Città del Vino, Cittàslow, Città del Bio, Città dei Sapori) almeno

la bellezza del paesaggio e la qualità del patrimonio storico-architettonico che non rappresentano solo attrattori importanti per i turisti ma sono in primis elementi a cui la comunità locale si sente particolarmente legata e riconosce come unici.

2 certificazioni riconosciute a Pitigliano. Al fine di poter valutare il grado effettivo di conoscenza dei residenti riguardo alle certificazioni ne sono state inserite alcune non conferite a Pitigliano (Cittaslow, Città del Bio e Città dei Sapori).

Città del tufo (25\%) e Bandiera Arancione (18\%) sono state le certificazioni più citate.

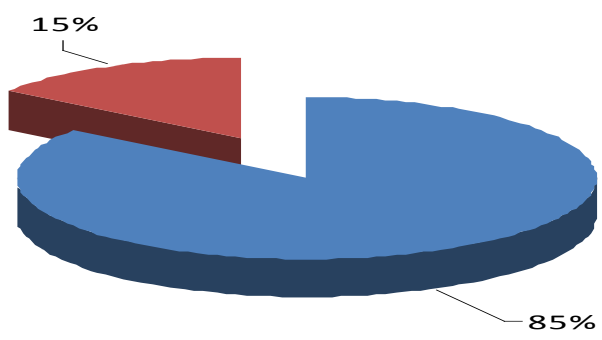

Sì, ne sono a conoscenza

- No, non ne sono a conoscenza

Figura 8 Conoscenza delle certificazioni conferite a Pitigliano. Fonte: ns elaborazione 
In riferimento a quale elemento secondo i residenti viene conferita una certificazione, il $39 \%$ individua nella qualità il fattore determinante, seguono la bellezza del paesaggio per il $31 \%$ e il valore del patrimonio culturale (21\%). Nuovamente qualità e bellezza sono fattori capaci di dare valore aggiunto e di accrescere la competitività di un territorio.

Per quanto riguarda la valutazione degli effetti delle certificazioni sul territorio agli intervistati è stato chiesta una valutazione generale e una valutazione puntuale inerente: al miglioramento dell'immagine del territorio, la congestione del traffico, l'incremento dello sviluppo economico e l'inquinamento amientale.

Il $92 \%$ dei residenti ritiene in generale le certificazioni possibili strumenti capaci di creare vantaggi socio-economici per il territorio e solo l'8\% sostiene che non hanno prodotto alcun vantaggio.

In riferimento alla valutazione puntuale agli intervistati è stato chiesto di attribuire un voto (da 1 a 10 ) circa la loro percezione sull'impatto che le certificazioni hanno Figura 9 Aree di intervento per rendere Pitigliano una destinazione turistica maggiormente competitiva. Fonte: ns avuto sui temi sopra elaborazione descritti.

Per quanto riguarda l'impatto della certificazione nel promuovere l'immagine del borgo l'89\% degli intervistati attribuisce un voto compreso tra 6 e 10 e solamente l'11\% attribuisce un voto insufficiente alla certificazione come strumento di comunicazione.

Il $70 \%$ (voto da 6 a 10) dei residenti ritiene che le certificazioni, aumentando la visibilità turistica, abbiano determinato un aumento del traffico, infatti gli intervistati evidenziano come il traffico sia maggiormente congestionato durante i periodi dell'anno con maggiore affluenza turistica.

Il $59 \%$ degli intervistati attribuisce un voto compreso tra 6 e10 all'impatto che le certificazioni hanno avuto sullo sviluppo economico locale, mentre il $41 \%$ ritiene le certificazioni strumenti non idonei a promuovere ricadute economiche sul territorio (voto da 1 a 5)

Per quanto riguarda l'impatto delle certificazioni sull'ambiente l' $81 \%$ dei residenti attribuisce un voto tra 1 e 5 cioè ritiene che nonostante un aumento dei flussi turistici non si evidenzia un aumento dell'inquinamento ambientale.

In sintesi emerge un quadro in cui le certificazioni sono in generale conosciute ed apprezzate dai residenti. Gli intervistati percepiscono le certificazioni come strumenti di comunicazione capaci di aumentare la visibilità e la notorietà del borgo, ma si evidenzia la difficoltà, da parte dei residenti, di individuare nella certificazione uno strumento in grado di favorire ricadute economiche capaci di attivare reale sviluppo locale. Infatti come già rilevato in altre analisi quantitative, con particolare riferimento al lavoro svolto sui borghi della regione Marche (Lorenzini, Calzati, Giudici, 2011) ${ }^{7}$, si conferma la difficoltà di evidenziare impatti positivi delle certificazioni sullo sviluppo locale.

\section{Qualità del territorio e turismo prospettive di sviluppo}

Nell'ultima sezione del questionario si domanda ai residenti di esprimere un giudizio negativo o positivo nei confronti delle azioni svolte dall'amministrazione locale al fine della valorizzazione del borgo. Per il 64\% degli intervistati i provvedimenti attuati dall'amministrazione locale non sono stati soddisfacenti ed adeguati al bisogno reale del territorio.

Sono state individuate tre aree importanti di intervento per incrementare la competitività turistica di Pitigliano: lo sviluppo di infrastrutture e trasporti, lo sviluppo di nuove offerte turistiche e il maggiore coinvolgimento del turista.

Il 50\% dei residenti ha individuato nello sviluppo di infrastrutture e trasporti un intervento fondamentale per la competitività turistica del territorio. Il 30\% degli intervistati considera una maggiore attenzione nel coinvolgimento del turista nella vita del borgo un rilevante fattore capace di aumentare la capacità competitiva di Pitigliano. Il 20\% degli intervistati ha individuato nello sviluppo di nuove offerte turistiche una possibile leva competitiva (figura 9).

Infine si è voluto chiedere ai residenti in quali ambiti del territorio l'amministrazione locale dovrebbe intervenire per migliorare la qualità della vita nel borgo. I residenti dovevano individuare, in un elenco, tre iniziative o

Il 23\% dei residenti ha individuato nella realizzazione di nuovi parcheggi l'ambito prioritario su cui intervenire in quanto i parcheggi esistenti non sono in grado di soddisfare le esigenze di residenti e dei fruitori del terriambiti di azione ritenuti prioritari (tabella 1). 


\begin{tabular}{|l|l|c|}
\hline \multicolumn{3}{|c|}{$\begin{array}{l}\text { In quali ambiti l'amministrazione comunale potrebbe implementare le } \\
\text { risorse al fine di migliorare la qualità della vita nel suo territorio } \\
\text { (max 3 ambiti) }\end{array}$} \\
\hline Ambiti & Numero & $\%$ \\
\hline Cura del centro storico & 53 & 13,5 \\
\hline Parchi e aree verdi & 30 & 7,6 \\
\hline Nuovi parcheggi & 91 & 23,2 \\
\hline Cura e tutela del patrimonio storico-architettonico & 25 & 6,4 \\
\hline Tra sporto pubblico & 59 & 15,0 \\
\hline Attività ricreative per anziani & 15 & 3,8 \\
\hline Attività ricreative per giovani & 43 & 11,0 \\
\hline Asili nido/scuole ma terne & 4 & 1,0 \\
\hline Attività commerciali & 18 & 4,6 \\
\hline Offerta culturale & 21 & 5,3 \\
\hline Raccolta rifiuti & 30 & 7,6 \\
\hline Altro & 4 & 1,0 \\
\hline Totale & 393 & 100,0 \\
\hline
\end{tabular}

rappresentate una innovativa leva competitiva nei territori minori. Inoltre per l'89\% dei cittadini la certificazione è riconosciuta come strumento di promozione capace di aumentare la visibilità del borgo a conferma di quanto evidenziato in letteratura.

Le brevi riflessioni sulla base dell'attività svolta evidenziano come i borghi, ritenuti territori minori, caratterizzati da produzioni di eccellenza, dalla bellezza del patrimonio storico-artistico e del paesaggio e dalla qualità dell'offerta locale, possano dare origine all'interno dei territori regionali a delle "distinct areas" in cui lo sviluppo turistico è il risultato di una nuova esperienza di fruizione del territorio. Ma soprattutto si evidenzia la capacità di tali territori di promuovere benessere per la comunità locale attraverso percorsi di sviluppo che pongono al centro non più la competitività ma l'abitabilità $\mathrm{e}$ la qualità del territorio.

In tali territori, in una logica post fordista, "la ricerca di ritmi differenti e di paesaggi da abitare entro i quali immaginare diversi stili di vita può diventare motivo di un agire collettivo, un progetto

torio, soprattutto nel periodo estivo quando vi è la maggior concentrazione di flussi turistici. Il 13\% degli intervistati ritiene prioritari interventi finalizzati alla cura del centro storico che come abbiamo evidenziato è il luogo che rappresenta per la comunità locale, maggiormente l'identità di Pitigliano. I trasporti costituiscono ulteriore punto di debolezza del territorio infatti il 15\% dei residenti destinerebbe risorse a disposizione della pubblica amministrazione nel miglioramento e nello sviluppo del servizio di trasporti pubblici non ritenuti adeguati alle esigenze dei cittadini

\section{Conclusioni}

I risultati dell'indagine confermano l'importanza del capitale sociale, il 54\% dei cittadini continua a vivere nel borgo per motivi legati alle relazioni familiari e sociali e del ruolo del patrimonio culturale in cui il $67 \%$ dei residenti si identifica con particolare attenzione al centro storico luogo in cui si sono sviluppate le prime relazioni sociali che hanno continuato a stratificarsi e consolidarsi nel tempo.

Pertanto come già evidenziato elementi intangibili quali l'identità, il patrimonio culturale e il capitale sociale costituiscono la matrice identitaria e l'armatura culturale del territorio (Carta, 2002). Per quanto riguarda le certificazioni territoriali il $70 \%$ dei residenti individua nella qualità e nella bellezza gli elementi fondamentali per la loro assegnazione pertanto tali fattori sono in grado se adeguatamente valorizzati di creare valore aggiunto e di politico-sociale in grado di giustificarsi con i propri risultati e la propria positività" (Lancerini, 2005, 17).

Il presente lavoro rappresenta un punto di partenza che lascia spazio ad integrazioni con altri strumenti, quali ad esempio la somministrazione di questionari agli amministratori locali dei piccoli centri, al fine di comprendere le problematiche e le opportunità dei territori minori in rapporto, non solo al flusso turistico, ma alla pianificazione dello sviluppo del territorio. Ulteriore approfondimento potrebbe derivare dalla somministrazione di questionari ai turisti, con lo scopo di valutare se e quanto è percepita la qualità della destinazione e di comprendere se le certificazioni sono realmente strumenti capaci di aumentare l'appeal e la visibilità di una destinazione.

Pertanto il presente lavoro costituisce una prima riflessione sul ruolo dell'identità, del capitale territoriale e delle certificazioni territoriali per uno sviluppo turistico locale orientato alla qualità, contribuendo al dibattito in corso che vuole, con l'apertura del ciclo della soft economy e della green economy, individuare nei territori minori non luoghi ai margini, ma aree capaci di attivare un modello di sviluppo sostenibile, endogeno e durevole.

\section{Ringraziamenti}

Si ringraziano gli studenti, dell'insegnamento di Marketing e Management del Territorio a.a. 2010/11 del Corso di Laurea in Economia e Sviluppo Territoriale della Facoltà di Economia dell'Università degli Studi di Siena, per il supporto dato nella somministrazione dei questionari. 


\section{Bibliografia}

Bagnasco A., Piselli F., Pizzorno A., Trigilia C.

2001 Capitale sociale: istruzioni per l'uso. Bologna: Il Mulino

Blain C., Levy S.E., Ritchie J.R.B.

2005 "Destination Branding” Journal of Travel Research, 43 (4): 328-338

Bonomi A., Rullani E.

2005 Il capitalismo personale. Vite al lavoro. Torino: Einaudi

Buhalis D.

2000 "Marketing the competitive destination of the future" Tourism Management, 21 (1): 97-116

Calzati V.

2009 "Territori lenti: definizioni e caratteri". In Aa.Vv. Sviluppo turistico e territori lenti (pp. 15-30). Milano: FrancoAngeli

Calzati V.

2011 "Territori lenti: nuove traiettorie di sviluppo". In V. Calzati, P. de Salvo, E. Nocifora (a cura di) Territori lenti e turismo di qualità. Prospettive innovative per lo sviluppo di un turismo di qualità (pp. 59-68). Milano: Franco Angeli

Capuano G.

2007 Mesoeconomia, Teorie ed evidenze empiriche di economia regionale. Milano: Franco Angeli

Carbone G., Fava U., Maggi M.

2000 Certificare il territorio per un turismo di qualità. Torino: IRES Piemonte

Carta M.

2002 L'armatura culturale del territorio. Il patrimonio culturale come matrice di identità e strumento di sviluppo. Milano: Franco Angeli

Coleman J.

1990 Foundations of Social Theory. Cambridge Masschussetts: The Belknap Press of Harward University Press

Commisione Europea

2010 Survey on the attitudes of Europeans towards tourism. Analytical report. Wave 2. Flash Eurobarometer n. 291

Fukuyama F.

1995 Trust. The Social Virtues and the Creation of Prosperity. New York: Free Press

Fukuyama F.

1996 Fiducia. Milano: Rizzoli

Garofoli G. e Mazzoni R.

1994 Sistemi produttivi locali: struttura e trasformazione. Milano: Franco Angeli

Golinelli C.M., Simoni M.

2006 "Gli strumenti del marketing turistico e territoriale". Sinergie Rapporti di ricerca, 2 (23): 61-78

Grahman B., Ashworth G.J., Tunbridge J.E.

2000 A Geography of Heritage: Power, culture and econo$m y$. London: Arnold

Granovetter M.

1985 Economic Action and Social Structure: The Problem of Embeddedness. American Journal of Sociology, 91
(3): 481-510

2009 Indagine sui visitatori attuali e potenziali dei borghi caratteristici. A cura della Doxa con la collaborazione della Mercury

2009 Indagine sui visitatori attuali e potenziali dei borghi. Risultati di un'analisi qualitativa. A cura della Doxa con la collaborazione della Mercury

Jacobs, J.

1961 The death and life of great American cities. New York: Vintage Book

Kahneman D. Krueger A.B.

2006 "Development in the Measurement of Subjective Well.Being”. Journal of Economic Perspectives, 20 (1): 3-24.

Kavarantzis M.

2005 "Place branding" The Marketing Review, 5 (4): 329342

Keller P.

1998 Destination Marketing, Publication, of the AIEST, vol. 40

Kotler P., Bowen J., Makens J.

2007 Marketing del turismo. Milano: McGraw Hill

Lancerini E.

2005 "Territori lenti: contributi per una nuova geografia dei paesaggi abitati italiani”. Territorio, .34: 9-15.

Lanzani A.

2005 "Geografie, paesaggi, pratiche dell'abitare e progetti di sviluppo". Territorio 34: 19-36

Layard R.

2006 Happiness. Lessons from a new science. London: Penguin

Lorenzini e., Calzati V., Giudici, P,

2011 "Territorial brands for tourism development: a statistical analysis on the Marche region". Annals of Tourism

Research, 38 (2): 540-560

Maggi M., Falletti V.

2000 Gli ecomusei. Cosa sono cosa potrebbero diventare. IRES Piemonte

Martini U.

2005 Management dei sistemi territoriali. Torino: Giappicchelli

Martini U., Ejarque j.

2008 Le nuove strategie di destination marketing. Milano : Franco Angeli

Mazzoli L.

2007 Turisti per casa. Milano: Franco Angeli

Mc Kercher B. e du Cros H.

2002 Cultural Tourism. New York: The Haworth Press

Mutti A.

1998 Capitale sociale e sviluppo. La fiducia come risorsa. Bologna: Il Mulino

Pichierri A.

1995 "Stato e identità economiche regionali". Stato e mercato, 44: 213-229

Pine B.J., Gilmone J. H.

2000 L'economia delle esperienze. Oltre il servizio. Milano: Etas 
Prideaux B., Cooper C.

2003 "Marketing and destination growth". Journal of Vacation Marketing, 9 (1): 35-51

Putnam R.

1993 La tradizione civica nelle regioni italiane. Milano: Mondadori

Richards G.

2001 Cultural Attractions and European Tourism. Wallingford: CAB International

Ritchie J.R.B.

1993 "Crafting a Destination Vision: Putting the Concept of Resident-Responsive Tourism into Practice". Tourism Management. 4 (5)

Rullani, E.

2003. Complessità sociale e intelligenza localizzata. In G. Garofoli Impresa e territorio (pp. 85-130). Bologna: Il Mulino

Santagata W.

2009 Libro bianco sulla creatività. Per un modello italiano di sviluppo. Milano: Egea

Sen A.

1999 Development as Freedom. Oxford: Oxford University Press

Shmelev S., Rodriguez-Labajos B.

2009 Dynamic multidimensional assessment of sustainability at the macro level: The case of Austria. Ecological Economics, 68 (10): 2560-2573

Stiglitz J., Sen A., Fitoussi J.

2009 Report by the Commission on the Measurement of Economic Performance and Social Progress.

Timothy D., Boyd S.

2003 Heritage Tourism. Prentice Hall

Trigilia C.

1999 "Capitale sociale e sviluppo locale". Stato e mercato, 57: 419-440.

Tunbridge J., Ashworth G.J.

1996 Dissonant Heritage: the management of the past as a resource in conflict. Chichester: Wiley

\section{Sitografia}

www.stradavinimaremma.it www.wikipedia.org www.comune.pitigliano.gr.it www.lamaremma.info www.lamaremmafabene.it www.naturalmenteitaliano.it www.borghitalia.it www.bandierearancioni.it
Allegato 1 Scheda delle risorse culturali ed ambientali del comune di Pitigliano

\section{Cultura (88)}

Beni storici architettonici-archelogici: (48)

1. Sinagoga di Pitigliano

2. Chiesa di Santa Maria e San Rocco

3. Duomo di Pitigliano

4. Oratorio del Santissimo Crocifisso

5. Chiesa di Sant'Antonio

6. Chiesa di San Rocco

7. Oratorio Rupestre

8. Chiesa di Santa Maria Assunta

9. Chiesa di San Michele

10. Chiesa della Madonna del Fiore

11. Cimitero ebraico

12. Cappella dei Santi Apostoli Giacomo e Filippo

13. Santuario della Madonna delle Grazie

14. Chiesa di San Francesco

15. Convento di San Francesco

16. Cappella di San Lorenzo

17. Chiesa di San Paolo della Croce

18. Cappella di Pian di Morrano

19. Chiesa di Santa Maria in Vinca

20. Acquedotto Mediceo

21. Fontana delle sette cannelle

22. Fontane gemelle

23. Palazzo Orsini

24. Pozzo di Palazzo Orsini

25. Teatro Salvini

26. Palazzo Comunale

27. Palazzo Lucci-Petruccioli

28. Palazzo Incontri

29. Palazzo della Pretura

30. Palazzo di Giustizia

31. Ex Ospedale di Pitigliano

32. Ospedale dei Poveri

33. Palazzo Vignoli

34. Scrittoio delle Fortezze

35. Palazzo Bruscalupi

36. Palazzo Santelli

37. Palazzo del Monte di Pietà

38. Porta di Parco Orsini

39. Fattoria del Piano

40. Fattoria di Terralba

41. Fattoria di Pian di Morrano

42. Porta di Sovana

43. Porta della Cittadella

44. Parco Orsini

45. Vie cave

46. Statonia

47. Monumento Alla Progenie Ursinea

48. Parco archelogico del Tufo

Musei e teatri: (8)

1. Museo Ebraico

2. Museo Archeologico Alberto Manzi

3. Museo civico archeologico 
4. Museo Palazzo Orsini

5. Museo Della Civiltà Giubbonaia

6. Centro d'Introduzione al Territorio

7. Museo Diocesano di Arte Sacra

8. Teatro Salvini di Pitigliano

\section{Eventi: (10)}

Eventi artistici:

1. Petilia Festival

2. Maremma DOC Festival

3. ArtExpò

4. Mostra Internazionale di Arte Contemporanea Kunstgroup

Rivisitazioni storiche/eventi folkloristici:

5. Torciata di San Giuseppe

6. Festa della Contea Pitigliano

7. Passato Prossimo

8. Festa dell'Epifania

9. Tradizionali feste di fin anno

Mostre mercati/fiere:

10. Fiera annuale di Pitigliano

\section{Feste/Sagre: (5)}

Feste Religiose:

1. Festeggiamenti San Paolo della Croce

2. Festeggiamenti in onore di San Rocco

3. Festeggiamenti in onore di Maria santissima delle Grazie

4. Infiorata del Corpus Domini

Feste/Sagre produzioni tipiche:

5. Apertura Cantinelle

Prodotti Tipici: (9)

1. Sfratto di Pitigliano

2. Frittelle di San Giuseppe di Pitigliano

3. Torsetto con la bolla di Pitigliano

4. Tortello dolce di Pitigliano

5. Tozzetto di Pitigliano

6. Cialdino dei Tufi

7. Focaccia bastarda di Pitigliano

8. Focaccia di Pasqua di Pitigliano

9. Migliaccia di Pitigliano

Prodotti Certificati: (8)

1. Vino Bianco DOC di Pitigliano

2. Olio extravergine di oliva Toscano IGP

3. Prosciutto Toscano DOP

4. Mortadella Bologna IGP

5. Pecorino Romano DOP

6. Pecorino Toscano DOP

7. Salamini Italiani alla Cacciatora DOP

8. Vitellone Bianco dell'Appennino Centrale IGP

Ambiente: (2)

Zone SIR- SIC- ZPS
Fiume Fiora

Biotopo Fiume Lente

Reti e Iniziative Intercomunali: (1)

1. Le strade del Vino e dei sapori Colli di Maremma

Certificazioni: (5)

1. Bandiera arancione

2. Borghi Più Belli d'Italia

3. A.N. Città del Vino

4. A.N. Città dell'Olio

5. Città del Tufo

\section{NOTE}

1 Si definisce identità l'insieme di specificità di un sistema territoriale, ricercate al fine di conseguire competitività nel confronto con gli altri territori e quindi la capacità di generare valore per i sovrasistemi e subsistemi di riferimento. Si definisce vocazione l'inclinazione naturale sociale, culturale ed economica di un territorio in ragione delle proprie componenti (Golinelli, 2002).

2 traduzione italiana di heritage nei documenti della Commissione Europea.

3 L'indagine è stata articolata in una fase quantitativa basata su interviste personali fatte ad un campione nazionale di 2106 italiani adulti (uomini e donne, di 15 anni ed oltre) e un indagine qualitativa effettuata attraverso 9 colloqui di gruppo (giovani dai 18 ai 35 anni, adulti dai 36 ai 55 e maturi dai 56 ai 70) di persone di Torino, Milano, Padova, Bologna, Roma, Napoli e Bari.

4 In alleato i risultati dell'analisi dettagliata del'offerta culturale e ambientale del Comune di Pitigliano.

5 Le denominazioni di origine e le indicazioni di provenienza geografica erano, agli inizi, semplici marchi collettivi tutelati da norme nazionali. Soltanto in seguito, grazie ad un adeguato riconoscimento in sede internazionale, sono stati giuridicamente riconosciuti anche al di fuori dei confini dello Stato nel quale venivano registrati. Il regolamento UE n. 2081/92 ha consentito la registrazione, in sede comunitaria, di numerose denominazioni di origine ed indicazioni di provenienza geografica nazionali per diversi prodotti agroalimentari, ponendo in essere, a favore di tali denominazioni ed indicazioni, una tutela completa, sotto il profilo giuridico, all'interno di tutti i Paesi della Comunità europea. $\mathrm{Al}$ riguardo si distinguono: DOC Denominazione di Origine Controllata)-DOCG Denominazione di Origine Controllata e Garantita)-IGT (indicazione Geografica Tipica)-IGP (Indicazione Geografica Protetta) -DOP (Denominazione di Origne Protetta).

6 Res Tipica è l'Associazione, costituita dall'ANCI- Associazione Italiana Comuni d'Italia- insieme alle Associa- 
zioni Nazionali delle Città di Identità, per la promozione delle identità territoriali italiane. Res Tipica nasce per salvaguardare e promuovere l'immenso patrimonio ambientale, culturale, turistico ed enogastronomico dei Comuni piccoli e medi del nostro Paese.

7 Ricerca orientata a valutare l'impatto del club di prodotto I Borghi più belli d'Italia nella Regione Marche. Un primo tentativo di analisi quantitativa è stato effettuato su un database costituito da 79 osservazioni costituite dai comuni considerati come turistici dalla Regione Marche e/o appartenenti al Club di Prodotto i Borghi più belli d'Italia con meno di 15.000 abitanti. L'individuazione di indicatori di qualità, di patrimonio, di sviluppo economico e di strutture ricettive hanno costituito la metodologia utilizzata. Tali analisi ha confermato la difficoltà di evidenziare impatti positivi della certificazione sullo sviluppo locale e di individuare indicatori capaci di misurare fattori come la qualità del patrimonio culturale e la capacità di fare sistema al fine di implementare la conoscenza dei fenomeni ai fattori immateriali e soggettivi 